\title{
RFID and ASP.NET based Campus Management System
}

\author{
Raghav Mehta \\ Student, Electronics and \\ Communication \\ Bhagwan Parshuram Institute \\ of Technology, GGSIPU \\ Delhi, India
}

\author{
Niranjan Bhattacharya, \\ Ph.D \\ HOD, Electronics and \\ Communication \\ Bhagwan Parshuram Institute \\ of Technology, GGSIPU \\ Delhi, India
}

\author{
Surbhi Goel \\ Student, Electronics and \\ Communication \\ Bhagwan Parshuram Institute \\ of Technology, GGSIPU \\ Delhi, India
}

\begin{abstract}
To create standards-based secure access to student's and employee's personal data, attendance records, student's mark sheets, expenditure and library management by using RFID tags and Web Service with the help of hardware kit, which synchronizes with one another. This system uses ASP.net to provide software interface and support standard Electronic Records of every individual in campus premises. Students and employees at campus can access their personal data, attendance records, mark sheets, books, payments (canteen, bills, and other fine) by using a single, all in one, card. Because the system is built on Web services it is easy to update, adapt and grow. This project uses the hardware kit to get the unique id. The hardware kit will send the employee and student EPC to the serial port of the system. Through allotted passwords the individual can have access to their account on system interface. With this system the college departments can improve the efficiency by enhancing the level of student's care while keeping the parents updated. Further a GSM modem is used to deliver a message, regarding daily presence of the student in the college, to their parents. Moreover incorporating GPS system with this project would help track the location of the students in case of emergencies.
\end{abstract}

\section{Keywords}

EPC, RFID card, GSM, GPS, KIOSK cash acceptors, Web services

\section{INTRODUCTION}

College campus consists of large number of individuals (employees and students) whose information and other records need to be maintained, which is somewhat a difficult task. Designing of campus management system based on RFID significantly reduces the efforts involved in management of records. The system not only can improve the work efficiency, student's performance and development, but also can save human and material resources. In recent years, RFID systems have been used in libraries for book identification, for self check-in, check-out. However, a one in all RFID card tackling all the aspects of campus has not been created yet. This super card will help students, employees in the campus to carry out different tasks with great ease, thus reducing time. RFID technology along with web service will help in establishing an organised system inside the campus. RFID is a combination of radiofrequency based technology and microchip technology. The Electronic product code (EPC) is read by Radio frequency technology regardless of item orientation or alignment. The web based software recognizes the unique id and then grants access to one's account. Figure 1 shows the necessary steps in order to pass the unique key and get access to the computer system. The card must be brought in the range of the reader. Why RFID? *Fastest, easiest, most efficient way to track, locate \& manage books, results, attendance, money * Efficient Book circulation management* Automatic Check-in and Check-out* Unique ID of the RFID tag prevents counterfeiting* Fast results display* Attendance management* Money control and expenditure*.

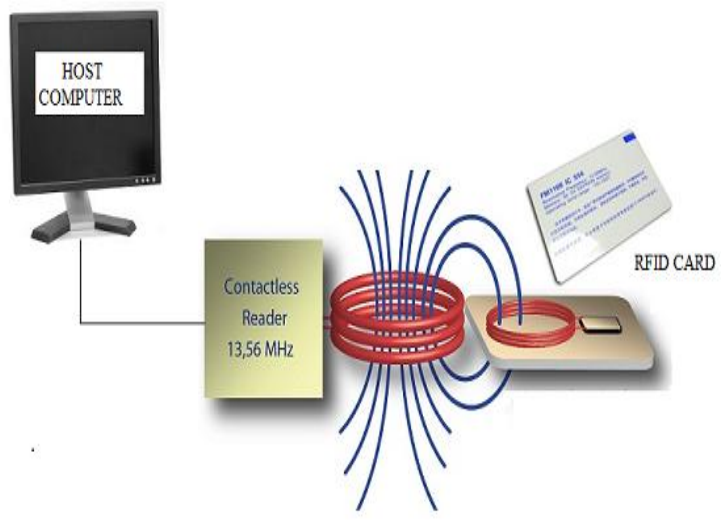

Fig. 1 RFID card interaction with module and finally accessing the software.

\section{MANAGEMENT AND FRAMEWORK}

This system provides the college to manage different department with great ease. Different management schemes have been provided.

\subsection{Attendance Management}

There are two methods provided for attendance updating. One is through the card, i.e. when an individual passes through a RFID card Reader (having high frequency), the attendance is automatically incremented by one. The timing and UID of that individual is noted by the system. The other method is by filling up the details manually on the software. The second method is provided to keep a track if there is mishandling. Both the students and employees can view their attendance. The professor can also view the attendance of the students, however cannot update it. The admin has the authority to update and define the leave that can be granted (medical and without medical).

\subsection{Library Management}

The system provides access to each and every book either through book name, author name or book UID. Every individual can check the availability, issue and return the book using his card. RFID tag will also be attached to each and every book to prevent book theft. The maximum limit to the 
number of books issued, fine (per book), and reissue limit is defined by the admin. The system sends messages to defaulters when the due date to return exceeds. The librarian can also generate slip of fine paid from the Individual's card and can collect it from the cash collector.

\subsection{Cafeteria Management}

The system provides the employee in the cafeteria to recharge card, make purchases, add and remove items and generate bills. He can also view his gross earning between a period of time specified by him. He is made aware by the system when the item finishes.

\subsection{Mark sheet Management}

The system provides every student to see his attendance and mark sheet on the system and thus reduce paper work. The data has to be entered by the data entry provider and keep it up to date. The professor can also view the marks of the student.

\subsection{Database Management}

The system allows to manage the database of the student, employee and book. Every individual will be having a card and a Unique Id corresponding to it. RFID tag will also be attached to each and every book to keep track on the books and its database.

\subsection{Security Management}

Security is one the most essential requirements for every project. It has been provided to different components of the system.

\subsubsection{Account security}

To ensure security of the individuals account, the admin is provided with the authority to reset password and account.

\subsubsection{Books security}

To protect book theft, the library exit gates can have high range RFID Card readers. If the book type shows 'issued' in the database, only then the book can be allowed to exit the library or else a siren is heard.

\subsubsection{Student Security}

A GSM device delivers message to the parents regarding the presence of the student in college on the basis of his attendance on the particular day. The college can also track the location of the individual in case of any emergency.

All in all the system provides complete management of every components (book, employee, student) present in the campus with a UID card assign to each components.

\section{APPLICATIONS}

The one in all cards or the RFID master card will completely change the working in the campus. It will improve the data storing and managing process. Attractive, useful and simple software provides user friendly interface to the user. This would not only help the students and the employee, but also the parents to judge and assess their child regularly. Finally, applying RFID products helps the institutions in automating money, book, attendance management, thus reducing overhead and minimizing class bunks, book thefts, etc. There are several users defined who can access the system.

\subsection{USERs}

Every user who wishes to access his account has to provide the RFID unique key by making contact of the card with the RFID module. Moreover the user also has to provide a password for his account. This provides further safety to the user in case of card theft. The default password will be the name of the card holder himself. He may change it after logging in. Figure 2 Shows how the user manages to get access to his account.

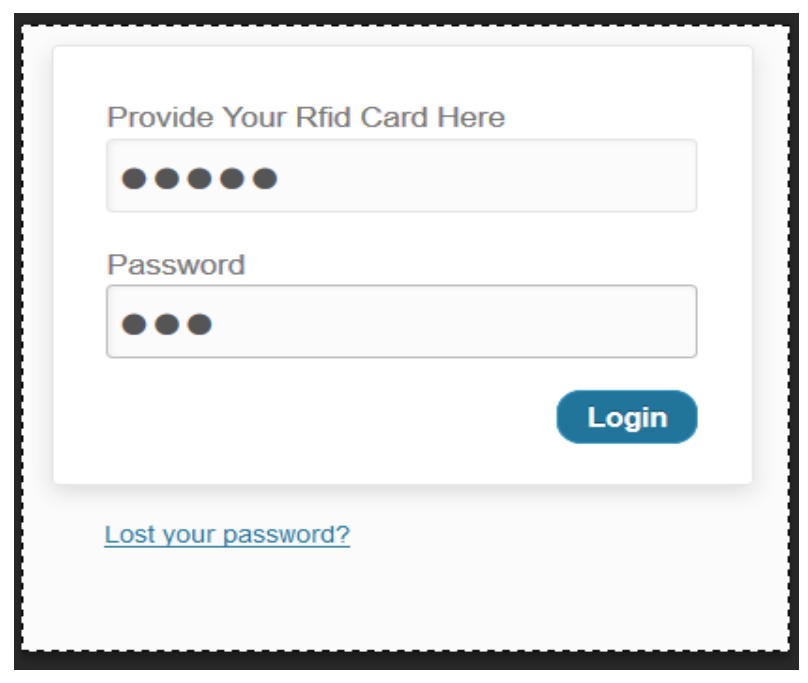

Fig. 2 Login process using UID key and Password

Since college consists of employees and students, both these have different authorities.

\subsubsection{Master Admin}

The admin has the authority to manage the whole software, add, remove, and edit databases. He can also edit and view the mark sheet and attendance. Moreover he can add course and corresponding subjects in the academics. The admin would be able to define the fine on the books and late payments. He can define total number of leaves that are granted with and without medical for both employees and student. Figure 3 shows the view for the master admin while accessing his account.

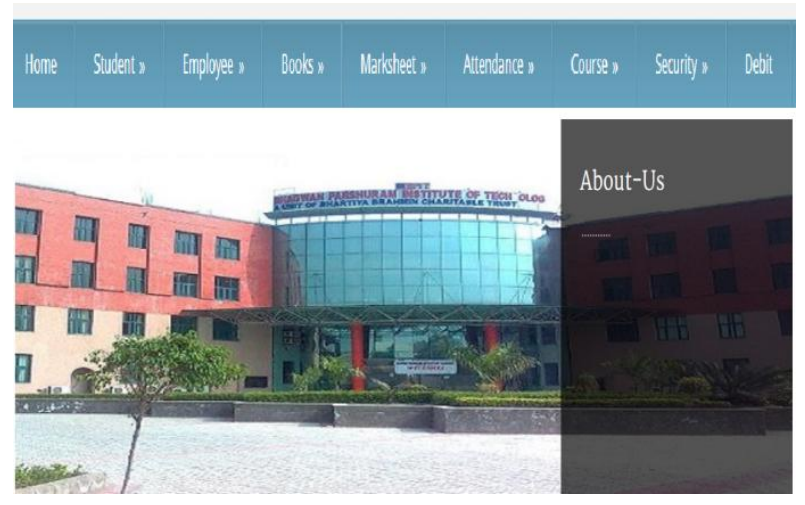

Fig. 3 Master administrator's account

The employees are divided on the basis of teaching on non teaching which subcategorizes them into respective roles. Teaching employees consists of professors and library department. While non teaching consists of admin, cafeteria, 
management, clerk, and ground-staff. Figure 4 shows the entry form of an employee.

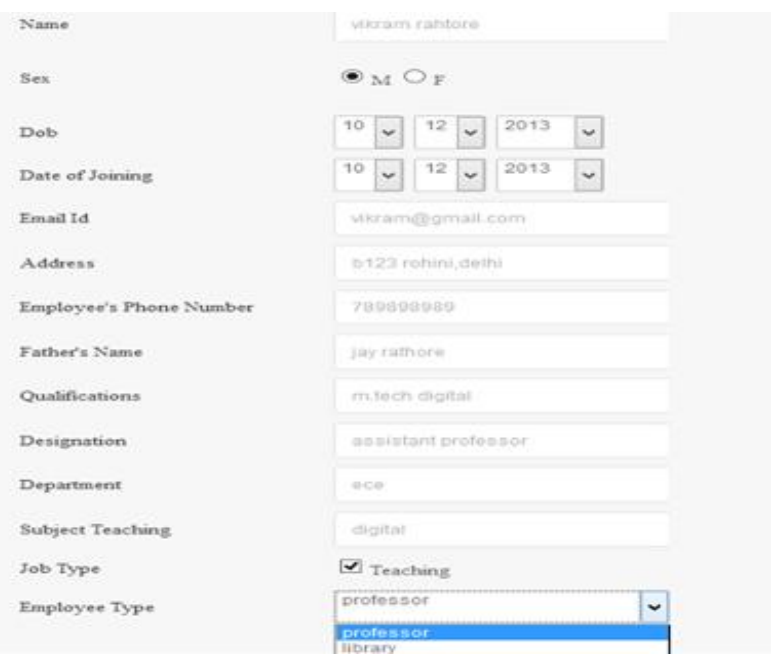

Fig. 4 Employee entry by Admin

\subsubsection{Employees}

Different employees have different authority on the basis of their role in the campus.

\section{i. Professor}

The professor is allowed to manage his own account and has access to student's marks and attendance. Figure 5 shows account and authorities of the professor while accessing student's attendance.

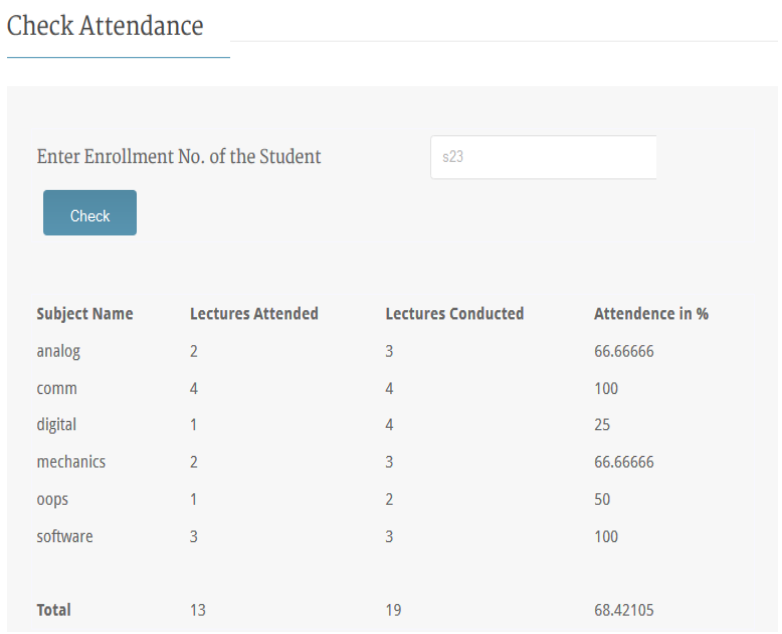

Fig. 5 student's attendance viewed by the professor

\section{ii. Librarian}

Although all the work for issue and return book is done automatically but the librarian account is provided to manage the books present in the library. He can look for the employees and student have been exceeded the due date to return books, $\mathrm{He}$ is also provided with an option to deliver message to the defaulters through the system (GSM provided). Moreover he can view the fine paid by the defaulters (fine paid through the card). Figure 6 shows the view for librarian.

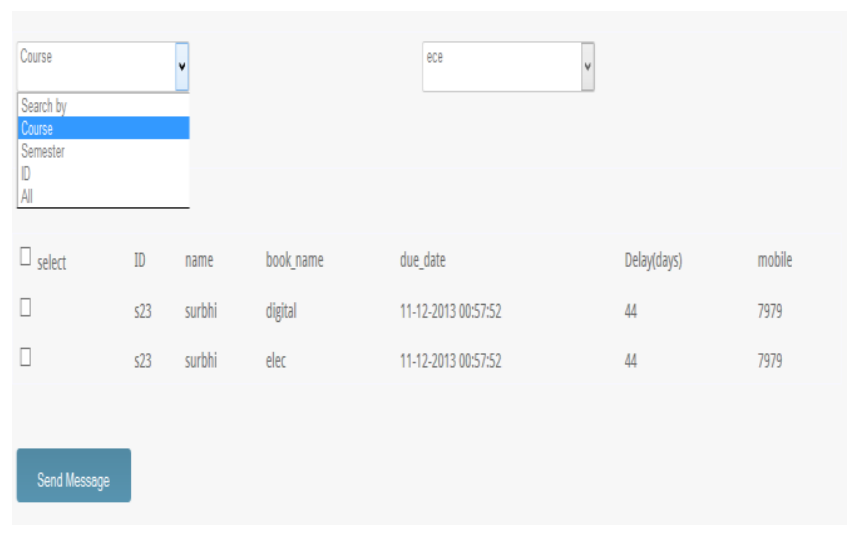

Fig. 6 librarian checking the defaulter and sending message

\section{iii. Cafeterias}

This employee can manage the canteen and has the authority to recharge the card and update balance. He has the authority to make purchases and generate bills. The software also provides the employee to know the gross earning, add and update items. Also, a message is displayed when item finishes. Figure 7 shows the authority given to the cafeteria employee while purchasing items.

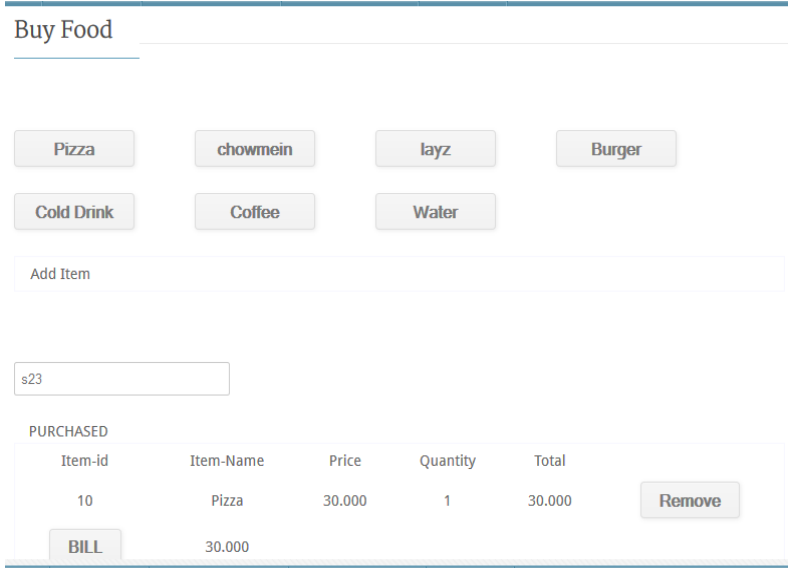

Fig. 7 purchasing items and generating receipts

iv. Others

Similar data abstraction has been done for, management, clerks and ground staff employees.

\subsubsection{Student}

Since students stand as the most important part of a campus system, simplest interface has been provided to them keeping in mind, data abstraction and authority. The student can access his account; view his updated attendance, marks, return and issue books. Since all the payments are done through the RFID card, the student can keep a record of his expenditure. Figure 8 shows student account while viewing his transaction details. 


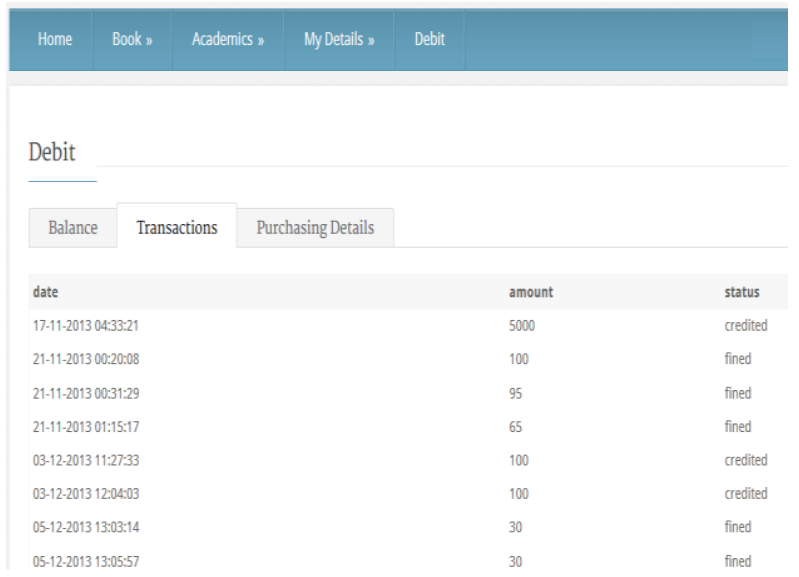

Fig. 8 student accessing his transaction details

\section{BENEFITS AND BARRIERS}

\subsection{Benefits}

RFID adoption in campus can not only reduce cost, paper work and provide simple managements, but also improve efficiency by tracking books and individual.

\subsubsection{RFID advantages over Barcode}

Earlier barcode system has been used to perform books issue and return. But this system is slow, tedious and time consuming. Barcode technology relies on optics, and therefore requires "line of sight," meaning that the barcode must be visible to the reader. Conversely, RFID tags can be invisible to the reader because they transmit information on demand using radio waves. A tag consists of a microchip applied to a miniaturized antenna, which carries a "unique tag ID" but can be programmed with other information. RFID offers advantages over barcode technology when:

- Invisible (or no line of sight) reading is required. For example, products within a box can be individually read or located with no line of sight to the reader.

- Multiple items must be read simultaneously.

- Read and write capability is required on the data carrier

\subsubsection{Time and Cost Saving}

This system reduces time. For example each Individual can purchase item by himself. Moreover there is no need to stand in line for issue and return of books. This can be done by every individual by himself. Other benefits include improving staff productivity, automated system, easy to access and simple interfacing. Further an automatic recharge machine (touch screen KIOSK cash payment acceptor HJL-3516) can be used which takes in money and updates the balance of the particular individual.

\subsubsection{Simple Interfacing}

When designing a software application user interface, the primary aspect to take into consideration is its general acceptance by end-users. After all, users would like to carry out their tasks as swiftly and simply as possible, and the user interface is just a tool to accomplish this goal. So, this software provides simple interface to the user (employee or student) in the campus to access all the necessary information.

\subsection{Barriers}

Two types of barriers have been detected.

\subsubsection{General}

RFID systems are not always reliable. Its reading accuracy depends on a variety of factors such as tagged object and placements, angle of rotation, and read distance. Also, Tag reliability can be impacted by humidity, metal surfaces, and more. Current RFID tags cannot withstand extreme temperatures without temperature-resistant environment.

\subsubsection{Software}

Attendance is incremented on the basis of how many times the individual's card enters in the range of RFID attendance reader. So, any other individual can make the attendance of any other person by just using their unique card. However we have also provided manual way to fill the attendance to prevent this kind of a barrier.

\section{CONCLUSION}

All the major barriers and benefits have been considered and a stabilized, user friendly and secure major interface has been made using RFID tags and ASP.net which provides easy managements. Considering the future developments, RFID technology will continue to yield larger memory capacities, wider reading ranges, and faster processing. RFID technology will not only be used for access control cards, tagging of returnable items but also for production and assembly tasks and thus will penetrate other market segments soon due to its lower costs and more functionalities.

\section{ACKNOWLEDGMENTS}

Our special thanks to the experts, Mr. Diwakar Garg, Dr. Pawan Whig, who have contributed towards development of this project.

\section{REFERENCES}

[1] Professional asp.net 3.5 in c\# and VB by Bill Evjen, Scott Hansel man, Devin Rader.

[2] Oehlmann, Dr. Heralds. "What is a smart label?" September 2002.

[3] Woods, J. "Strategic Planning SPA-23-1513: Prepare for Disillusionment with RFID." June 29, 2004

[4] ISO/IEC 18000-x. "RFID Air Interface Protocols." ISO/IEC 15961,2 "RFID Data Protocol." ISO/IEC 15963. "RFID Unique Tag ID." Valider, Guenther. Interactive L

[5] CURTIN, R. J. KAUFFMAN, AND F. J. RIGGINS Making the 'most' out of rfid technology:a research agenda for the study of the adoption, usage and impact of rfid, Information Technology and Management, 8, 2 (2006), 87-110 